\title{
Chapter 16 \\ Effect of Non-parallel Applicator Insertion on 2.45 GHz Microwave Ablation Zone Size and Shape
}

\author{
Austin W. White, Dwight D. Day, and Punit Prakash
}

\subsection{Introduction}

Microwave ablation (MWA) is an established modality for minimally invasive treatment of tumors in the liver and other organs. During MWA, a microwave antenna (also referred to as an ablation applicator) is inserted into the target tissue and radiates microwave power, which is absorbed by the surrounding tissue and leads to heating. Compared to other energy modalities for thermal ablation, MWA offers the advantage of rapid heating of large tissue volumes via the simultaneous use of multiple applicators [1]. The electromagnetic power absorbed within tissue during multiple applicator MWA is a function of the system operating frequency, tissue biophysical properties, applied power levels, radiation pattern of each applicator, and the relative spacing between the applicators. Preclinical experiments in ex vivo tissue are often used to characterize the size and shape of ablation patterns for specific devices, and these data may be used to assist in treatment planning [2]. Experimental studies to characterize multiple applicator ablation patterns typically employ the applicators inserted into the tissue sample in a parallel manner [1]. In clinical practice however, parallel applicator insertion may not be feasible due to anatomical constraints. As illustrated in [3], the spacing between applicator tips may be up to twice as much as the applicator spacing at the insertion point on the skin surface.

Studies investigating the impact of non-parallel applicator insertion during interstitial hyperthermia with microwave antenna arrays determined that skewed applicator placement shifted the location of the region of maximum power absorption [4]. However, prior simulation studies to assess ablation zones created by MWA with non-parallel applicator configurations were limited due to the use of models

\footnotetext{
A. W. White $(\bowtie) \cdot$ D. D. Day · P. Prakash

Department of Electrical and Computer Engineering, Kansas State University,

Manhattan, KS, USA

e-mail: prakashp@ksu.edu
} 
which did not incorporate temperature-dependent tissue dielectric properties [3]. Recent studies have highlighted the importance of incorporating temperaturedependent tissue dielectric properties in computational models of MWA [5].

This chapter summarizes the use of multi-physics computational models and experiments in ex vivo tissue to compare the size and shape of ablation zones with parallel and non-parallel applicators.

\subsection{Materials and Methods}

We implemented finite element method (FEM) computational models of microwave tissue heating to comparatively assess ablation profiles achieved with a pair of $2.45 \mathrm{GHz}$ interstitial dipole antennas spaced $10-20 \mathrm{~mm}$ apart. The antennas were inserted in parallel and non-parallel configurations. Experimental studies were conducted ex vivo using liver tissue for a subset of these configurations. Computational modeling results were compared against the experimental observations of ablation zone profiles.

\subsubsection{MWA Applicator Configurations}

We investigated nine different MWA applicator insertion configurations, including three cases with parallel applicator insertion and six cases with non-parallel applicator insertion. We considered inter-applicator spacings of 10-20 $\mathrm{mm}$ to capture the range of applicator spacing commonly encountered in clinical applications. As illustrated in Fig. 16.1, the parallel cases had antennas inserted into the liver with the center of the microwave applicators at separation distances denoted $D_{\text {ins. }}$. The non-parallel cases had the tip of each applicator moved toward the centerline by $2.5 \mathrm{~mm}, 5 \mathrm{~mm}$, or $7.5 \mathrm{~mm}$ each, resulting in tip spacings $\left(D_{\text {tip }}\right)$ of $15 \mathrm{~mm}, 10 \mathrm{~mm}$, or $5 \mathrm{~mm}$, respectively.

Fig. 16.1 Illustration of non-parallel MWA applicator insertion

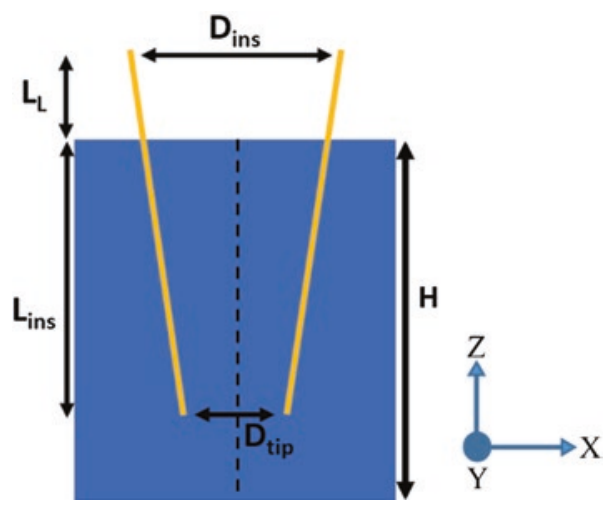


In Fig. 16.1, $D_{\text {ins }}$ is the nominal distance between the antennas and $D_{\text {tip }}$ is the distance between the tips of the antennas. For the parallel cases, $D_{\text {ins }}$ is equal to $D_{\text {tip }}$. For the non-parallel cases, $D_{\text {tip }}$ was changed in multiples of $5 \mathrm{~mm}$ (each antenna moved $2.5 \mathrm{~mm}$ ). The tip of each antenna was moved inward by half the total tip displacement, so that the setup was always symmetric with respect to the centerline. $L_{\text {ins }}$ is the distance between the antenna tip and the upper surface of the liver tissue. $L_{\text {ins }}$ corresponds to the length of the antenna inside the liver for a parallel case. The depth of the antenna tip from the top of the liver remained constant in all experiments. Therefore, the total length of the antenna in the liver slightly increases for a non-parallel case in order to maintain the same tip depth from the surface of the liver. $\mathrm{L}_{\mathrm{L}}$ was chosen to act as a reference to define the insertion distance.

For this study, the MWA applicators were uncooled, interstitial, dipole antennas operating at $2.45 \mathrm{GHz}$. The antennas were created using UT-85 coaxial cable with the distal tip of the cable modified as described by Curto et al. [6]. Each antenna was energized with $15 \mathrm{~W}$ applied power (at the antenna input). We chose this power to be within the clinically relevant range, while yielding ablation zone sizes not impacted by the limited size of the tissue samples used in our experiments.

As illustrated in Fig. 16.1, ablation zone extents were assessed primarily in the XZ-plane.

\subsubsection{Computational Model of Microwave Ablation}

Three-dimensional multi-physics computational models of microwave tissue heating were implemented using the FEM solver within COMSOL Multiphysics (v5.2a). Models were employed to compute the electric fields radiated into tissue and the subsequent heat transfer due to absorbed microwave power. The Helmholtz electromagnetic wave equation, Eq. 16.1, was used to calculate the electric field at all mesh points.

$$
\nabla \times\left(\mu_{\mathrm{r}}^{-1} \nabla \times \mathbf{E}\right)-\frac{\omega^{2}}{c_{0}^{2}}\left(\varepsilon_{\mathrm{r}}-\frac{i \sigma}{\omega \epsilon_{0}}\right) \mathbf{E}=0
$$

Here $\mu_{\mathrm{r}}$ is the relative permeability, $\varepsilon_{\mathrm{r}}$ is the relative permittivity and $\sigma$ is the electrical conductivity of the material, $c_{0}$ is the speed of light in free space, $\omega$ is angular frequency, and $\mathbf{E}[\mathrm{V} / \mathrm{m}]$ is the electric field vector. From the electric field, we can calculate how much power is absorbed within the tissue using Eq. 16.2, which serves as the heat source for the transient heat equation, Eq. 16.3.

$$
Q_{\mathrm{e}}=\frac{1}{2} \sigma|\mathbf{E}|^{2}
$$




$$
\rho c \frac{\partial T}{\partial t}=\nabla \cdot k \nabla T+Q_{\mathrm{e}}
$$

where $T$ is temperature $[\mathrm{K}], \rho$ is tissue density $\left[\mathrm{kg} / \mathrm{m}^{3}\right]$, and $C$ is the specific heat capacity of tissue $[\mathrm{J} /(\mathrm{kg} \mathrm{K})]$.

We did not include blood perfusion in our simulations as our ex vivo experimentation would also lack blood perfusion and the primary goal of this work was to compare simulation results with experimental measurements. The electrical properties of liver tissue at $2.45 \mathrm{GHz}$ were dynamically adjusted as a function of the liver temperature as described in [7]. The nominal value of tissue relative permittivity was $\varepsilon_{\mathrm{r}}=57.9$ and effective conductivity $\sigma=1.09 \mathrm{~S} / \mathrm{m}$ [8].

The applicators were inserted into a cuboidal volume of liver tissue (length and width of $60 \mathrm{~mm}$ and a height of $80 \mathrm{~mm}$ ). A non-uniform tetrahedral mesh was employed in the simulations. In our case, the finest resolution was within the antenna and coarsest resolution was used at distances further away from the applicator. The conducting elements in the antennas were approximated as perfect electric conductors. Electrical scattering and thermally insulating boundary conditions were imposed on the exterior surfaces of the liver and the insulating catheter.

\subsubsection{Experimental Assessment of Dual Applicator Microwave Ablation Zones}

\section{Instrumentation and Procedure}

Two insulated dipole antennas were fabricated according to the dimensions given in the paper by Curto et al. [6]. The antennas had an active dipole length of $10.9 \mathrm{~mm}$ and a gap of $1 \mathrm{~mm}$ and were tuned to operate at $2.45 \mathrm{GHz}$. These antennas did not have any active cooling. Antennas were fabricated using Micro-Coax UT-85, with a thin heat shrink tubing (3M, FP-301 26-28 AWG) acting as an insulating sheath. An SMA connector (Huber+Suhner) was attached to the end of the coax to form the connector. Antenna reflection coefficient measurements using a Vector Network Analyzer (VNA) verified that the antennas were properly matched $\left(S_{11}\right.$ of at least $-10 \mathrm{~dB}$ at the operating frequency) to the feeding transmission line.

For the experimental setup, a Flash Forge Creator Pro 3D printer was used to create a base from polylactic acid (PLA). The base was designed to hold the antennas and liver tissue during experimental testing. The internal measurements of the base were $60 \mathrm{~mm} \times 60 \mathrm{~mm} \times 85 \mathrm{~mm}$. The template blocks held the antennas in the same configurations as the simulations. The following antenna configurations were investigated experimentally: $20 \mathrm{~mm}$ parallel, $20 \mathrm{~mm}$ to $15 \mathrm{~mm}$ (converging), $15 \mathrm{~mm}$ parallel, and $15 \mathrm{~mm}$ to $10 \mathrm{~mm}$ (converging).

Ex vivo ablations were conducted in fresh bovine liver. Liver samples were procured from a local meat-processing plant and cut to fit into the base. The liver samples were placed in plastic bags and then warmed in a water bath (Polyscience 7306) 
until they reached a temperature of $\sim 30{ }^{\circ} \mathrm{C}$. Five experiments were performed for each configuration. For each experiment, one liver piece was removed from the water bath and placed into the base and the template block was then snapped into place. The antennas were inserted through the template block to a depth of $6 \mathrm{~cm}$ into the liver.

We applied a $30 \mathrm{~W}$ forward power signal at $2.45 \mathrm{GHz}$ (SAIREM microwave generator) and used an equal power splitter (Pasternack PE-T1000) to divide the power into two separate, phase-matched signals fed to each antenna. We then used two power meters (Bird Technologies 7022-1-02020 and Bird Technologies 5012D) to track the power level delivered to each antenna. We adjusted the total average forward power to remain close to $30 \mathrm{~W}$ during each experimental ablation.

The liver was sectioned in half after the ablation, revealing the ablation zone in the XZ plane. This was done by removing the template block along with the red connector piece and slicing the liver with a knife down the slot in the base as shown in Fig. 16.2. Both halves of the liver were then placed on a flatbed scanner (Epson V550 Photo Color Scanner) which was used to acquire a color (RGB) image of the ablation zone at 400 dpi. The scanner was used to prevent skew from photos that might appear in pictures taken with a hand-held camera. The scanner has the added benefit of keeping the lighting and resolution constant for all pictures, which is desirable for automated processing of acquired images.

\section{Image-Based Analysis of Experimental Ablation Zones}

The resulting pictures were processed using the basic image processing techniques available in Python 3.6 to measure the size and compare the shape of the resulting ablation zones. A basic thresholding operation in both the RGB color space as well as the Hematoxylin-Eosin-DAB (HED) color space (HED used often in detecting blood composition) was the primary method used to detect the ablated area of the

Fig. 16.2 3D printed base with template block in place to hold the antennas

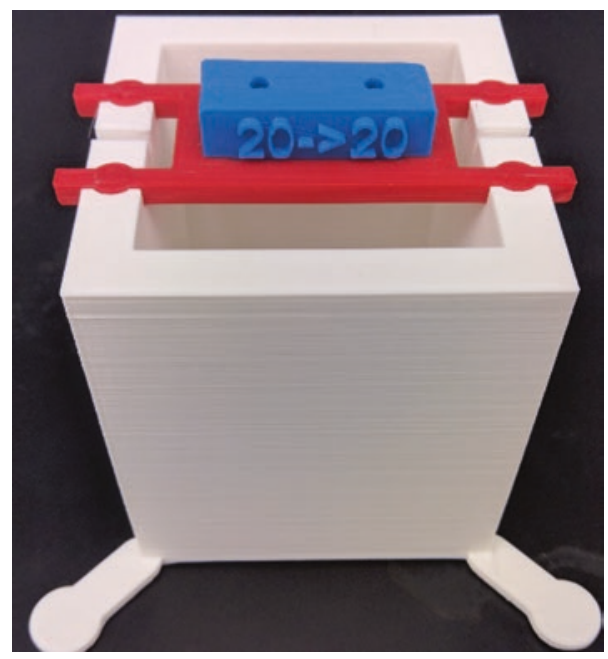


liver tissue. We used skimage to convert the RGB image into the HED color space. Then scipy was used to perform a small Gaussian blur $(\sigma=.19)$ on the resulting non-binary image to remove noise, and then converted back to a binary image. A series of morphological operators (erosion and then dilation using a disk of size 3 pixels) further reduced noise and smoothed the edges of the ablation region. After the morphological operators, the scipy function - binary fill holes - was used to fill in the holes in the middle of the ablated region. Skimage region properties were used to single out the ablation region, which corresponded to the largest region in the image after noise removal. An edge detection operation was used on the largest area to determine the size of the ablation zone. Further details of the image analysis procedure are provided in [9].

Once the shape of the ablation region was segmented in all ablations for a given scenario, they were overlaid for aggregate analysis. Each segmented ablation zone was centered and aligned to the same orientation as part of the overlay process. Region properties of the ablation zone were used to find the center of mass for each ablation area. We also applied a rotation on an image-by-image basis depending on the placement of the liver sample on the scanner bed. The angle of rotation was determined qualitatively for each of the ablation areas. After aligning the ablation shapes, we added the binary score ( 0 or 1$)$ for each pixel from multiple repetitions of each ablation scenario together to find the composite ablation image. Since we performed $n=5$ experiments for each experimental scenario, this composite image contained only integers with values that ranged between zero and five. The value of five occurred in regions where the tissue was ablated in each of the five experiments; likewise, the areas that had a value of one are locations where the tissue was ablated in only one of the five experiments. The darkest mass in the center corresponded to an ablation area in all experiments. From this, we found the maximum and minimum ablation shape for each scenario, as well as the $75 \%$ contour (i.e., the region that we would expect to see ablated in $75 \%$ of ablations). To find the maximum, we converted the image to a binary image format where only the highest number (five) would be a one, all else would be zero. A canny edge detection algorithm was then used to find the maximum shape.

We compared the shape of the ablation zone extents from experiments using the Dice Similarity Coefficient (DSC) metric. This metric is commonly used in image processing applications to compare shape similarity. The DSC is calculated using Eq. 16.4,

$$
\mathrm{DSC}=\frac{2|A \cap B|}{|A|+|B|}
$$

For the experiments, A and B are binary images that have been detected through image processing. Specifically, the areas inside some contours (typically the $75 \%$ contour). For the simulations, we use the $\mathrm{XZ}$ plane that bisects the liver and antennas and considered only points on that plane that are at or above $55^{\circ} \mathrm{C}$ as an estimate of the simulated ablation zone. We also employed a volumetric approach that compared the entire volume of the ablation region that is above $55^{\circ} \mathrm{C}$ to the $3 \mathrm{D}$ simulation results. 


\subsection{Results and Discussion}

Figure 16.3 illustrates experimental results for the ablation zone segmentation, alignment, aggregation, and averaging process. Table 16.1 lists Dice Similarity Coefficients between microwave ablation zones for antennas spaced 10-20 mm apart in parallel and non-parallel configurations.

Although both experiments and simulations predicted similar trends, experimentally measured DSCs were $\sim 15 \%$ lower. This discrepancy is possibly due to the jagged edges of experimental ablation zones, which confound image similarity metrics.

Figure 16.4 illustrates simulated and experimental ablation zones for $15 \mathrm{~mm}$ parallel and non-parallel (2.5 mm displacement of antenna tips) configurations. Both simulations and experiments indicated small changes in ablation zone shape. For the $20 \mathrm{~mm}$ parallel vs. non-parallel configurations illustrated in Fig. 16.5, the difference between ablation zone shapes is slightly more pronounced, as evidenced by the smaller DSC and observed ablation zone shapes. The presented method therefore provides a means for comparing ablation zone shapes for various configurations, in contrast to the widely used approach of comparing parameterized measurements of ablation zone geometries. This approach is especially helpful for analysis of shapes that are not close approximations of spheres/ellipses.
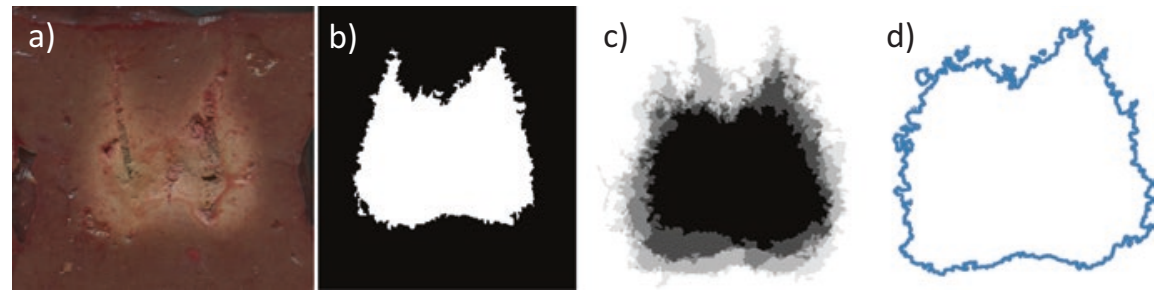

Fig. 16.3 Image processing at various stages- from left to right: (a) scanned image of ablation zone in ex vivo liver, (b) segmented ablation region, (c) overlay of segmented regions, and (d) the resulting $75 \%$ contour

Table 16.1 Dice Similarity Coefficients between microwave ablation zones

\begin{tabular}{l|l|l|l}
\hline Antenna spacing at insertion & Antenna tip spacing & DSC simulated & DSC experimental \\
\hline 10 & 5 & 0.95 & \\
\hline 15 & 10 & 0.93 & $0.81 \pm 0.003$ \\
\hline 15 & 5 & 0.89 & \\
\hline 20 & 15 & 0.85 & $0.73+0.06$ \\
\hline 20 & 10 & 0.78 & \\
\hline
\end{tabular}


A

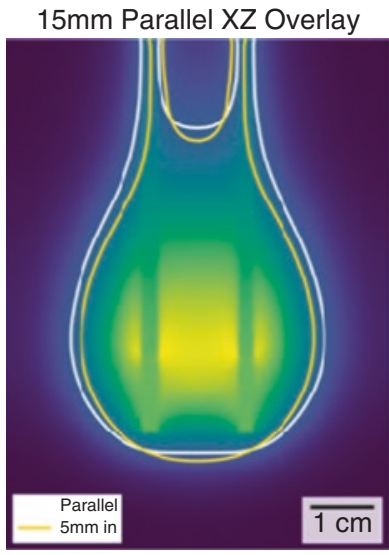

B

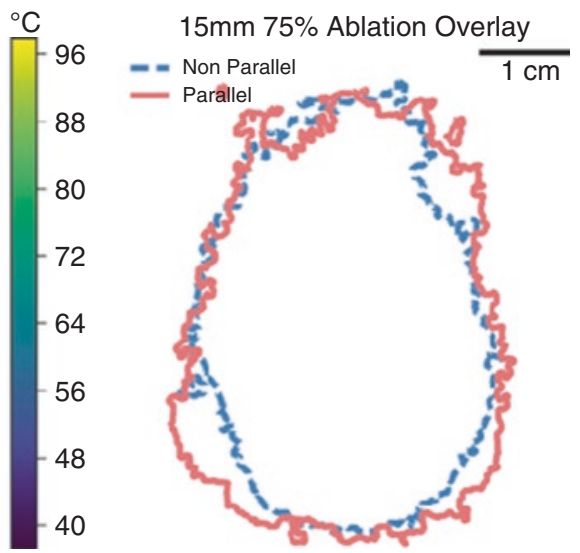

Fig. 16.4 (a) Illustration of simulated ablation zones for $15 \mathrm{~mm}$ parallel vs. non-parallel configurations; (b) $75 \%$ ablation overlay from experiments

A

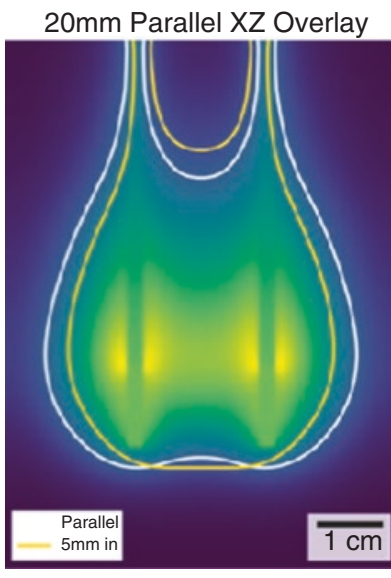

B

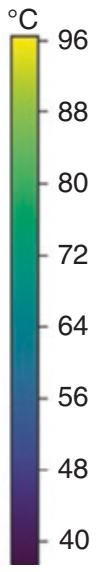
$20 \mathrm{~mm} 75 \%$ Ablation Overlay

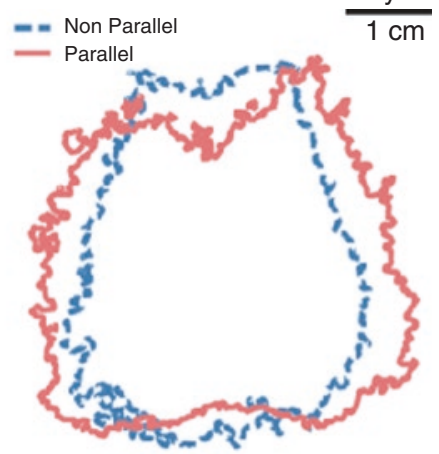

Fig. 16.5 (a) Illustration of simulated ablation zones for $20 \mathrm{~mm}$ parallel vs. non-parallel configurations; (b) $75 \%$ ablation overlay from experiments

\subsection{Conclusion}

The use of microwave ablation for treating tumors in the liver and other organs is growing. Clinicians who deploy multiple antennas for treating hepatocellular carcinoma (HCC) may not always be able to insert the antennas parallel to each other, and it is not yet known what impact this has on the ablation outcome. This research was an investigation to assess the potential change in ablation volume for non-parallel 
insertion. These results may serve as a guide to practitioners to determine the potential impact of non-parallel antenna insertion when conducting clinical procedures.

We found that non-parallel insertion could have a measurable change on the ablation size and volume for our setup. There was up to a $30 \%$ variability for some of the more severe cases of non-parallel insertions studied. We also found that the rate of change for ablation size does vary considerably and did not vary linearly with the distance of the tip displacement from the original parallel position.

The model we used for simulating ablation was relatively accurate at predicting the shape of the ablation zone for our experiment and was also capable of showing how the ablation shape would change given a small tip offset distance. The amount of similarity between the parallel and non-parallel insertions did vary when comparing the simulation and the experimentation, possibly due to unaccounted tissue shrinkage in the simulations.

Acknowledgments This research was funded in part through NIH grant R01 CA 218357.

\section{References}

1. Brace, C. L., Laeseke, P. F., Sampson, L. A., Frey, T. M., van der Weide, D. W., \& Lee, F. T. (2007). Microwave ablation with multiple simultaneously powered small-gauge triaxial antennas: Results from an in vivo swine liver model. Radiology, 244(1), 151-156.

2. Amabile, C., et al. (Feb. 2017). Microwave ablation of primary and secondary liver tumours: Ex vivo, in vivo, and clinical characterisation. International Journal of Hyperthermia: the Official Journal of European Society for Hyperthermic Oncology, North American Hyperthermia Group, 33(1), 34-42.

3. Mukherjee, S., Curto, S., Albin, N., Natarajan, B., \& Prakash, P. (2015). Multiple-antenna microwave ablation: Analysis of non-parallel antenna implants. Presented at the Energy-based Treatment of Tissue and Assessment VIII, vol. 9326, p. 93260U.

4. Clibbon, K. L., \& McCowen, A. (1993). Thermal modelling of nonideal interstitial microwave antenna array hyperthermia for the treatment of cancer. In 1993 IEEE MTT-S International Microwave Symposium Digest, Atlanta, pp. 1147-1150.

5. Deshazer, G., Hagmann, M., Merck, D., Sebek, J., Moore, K. B., \& Prakash, P. (2017). Computational modeling of $915 \mathrm{MHz}$ microwave ablation: Comparative assessment of temperature-dependent tissue dielectric models. Medical Physics, 44, 4859.

6. Curto, S., Taj-Eldin, M., Fairchild, D., \& Prakash, P. (2015). Microwave ablation at $915 \mathrm{MHz}$ vs $2.45 \mathrm{GHz}$ : A theoretical and experimental investigation: Microwave ablation at $915 \mathrm{MHz}$ vs 2.45 GHz. Medical Physics, 42(11), 6152-6161.

7. Ji, Z., \& Brace, C. L. (2011). Expanded modeling of temperature-dependent dielectric properties for microwave thermal ablation. Physics in Medicine and Biology, 56(16), 5249-5264.

8. IT'IS Foundation. (2015). “Tissue Properties Database V3.0.” IT'IS Foundation.

9. White, A. (2018). Effect of non-parallel applicator insertion on microwave ablation zone size and shape. 
Open Access This chapter is licensed under the terms of the Creative Commons Attribution 4.0 International License (http://creativecommons.org/licenses/by/4.0/), which permits use, sharing, adaptation, distribution and reproduction in any medium or format, as long as you give appropriate credit to the original author(s) and the source, provide a link to the Creative Commons license and indicate if changes were made.

The images or other third party material in this chapter are included in the chapter's Creative Commons license, unless indicated otherwise in a credit line to the material. If material is not included in the chapter's Creative Commons license and your intended use is not permitted by statutory regulation or exceeds the permitted use, you will need to obtain permission directly from the copyright holder.

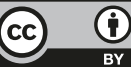

